\title{
Radical inguinal orchidectomy: the gold standard for initial management of testicular cancer
}

\author{
Samantha G. Koschel $^{1,2}$, Lih-Ming Wong ${ }^{1,2}$ \\ ${ }^{1}$ Department of Urology, St. Vincent's Hospital Melbourne, Melbourne, Australia; ${ }^{2}$ Department of Surgery, University of Melbourne, Melbourne, \\ Australia \\ Contributions: (I) Conception and design: All authors; (II) Administrative support: SG Koschel; (III) Provision of study material or patients: None; \\ (IV) Collection and assembly of data: None; (V) Data analysis and interpretation: None; (VI) Manuscript writing: All authors; (VII) Final approval of \\ manuscript: All authors. \\ Correspondence to: Dr. Samantha G. Koschel. St. Vincent's Hospital Melbourne, 41 Victoria Pde Fitzroy VIC 3065, USA. Email: sgkoschel@gmail.com.
}

\begin{abstract}
Radical inguinal orchidectomy with division of the spermatic cord at the internal inguinal ring is the gold standard for diagnosis and local treatment of testicular malignancies. The technique is well established and described in detail in this paper, collating methods from various surgical textbooks and articles. We also discuss pre-operative considerations including fertility counselling and potential testicular prosthesis at time of orchidectomy, and the importance of contemplating differential diagnoses such as para-testicular sarcoma and primary testicular lymphoma (PTL) prior to performing radical orchidectomy (RO). The evidence and indications for new surgical techniques to treat local testicular malignancies are also described, including testis sparing surgery (TSS) and spermatic cord sparing orchidectomy.
\end{abstract}

Keywords: Testicular neoplasms; testicular cancer; orchiectomy; testis sparing surgery (TSS)

Submitted Jun 26, 2019. Accepted for publication Sep 04, 2019.

doi: $10.21037 /$ tau.2019.12.20

View this article at: http://dx.doi.org/10.21037/tau.2019.12.20

\section{Introduction}

Testicular cancer is considered a relatively rare malignancy, representing $1 \%$ of cancer in males and $5 \%$ of urological neoplasms overall. However, it remains the most common solid organ malignancy in young men aged 20-40, with approximately 3-10 new cases diagnosed each year per 100,000 men in Western societies (1). This equates to an estimated 9,500 new diagnoses and 400 deaths per year in the United States (2). Incidence is increasing, particularly in Caucasian and Hispanic populations, but a trend toward earlier stage at diagnosis is observed, likely reflective of improved awareness (3).

The histological classification of testicular cancer is predominantly germ cell tumour (GCT) in up to $95 \%$ of cases, of which the vast majority are derived from germ cell neoplasia in situ (GCNIS) (1). GCT are then further classified into seminoma and non-seminomatous GCT
(NSGCT), with seminoma accounting for $52-56 \%$ and NSGCT for $44-48 \%$ (4). The predominant subtypes of NSGCT are embryonal carcinoma, yolk sac tumour, choriocarcinoma, teratoma and mixed GCT which can include a combination of any subtype. The remaining 3-6\% of adult testicular tumours are sex cord/stromal tumours with less than $10 \%$ of these following a malignant course (5).

Inguinal exploration with radical orchidectomy (RO) is the gold standard initial management of any testicular mass suspicious for malignancy $(6,7)$. RO establishes a histopathological diagnosis, facilitates staging of the disease, and is curative for $>80 \%$ of men with clinical stage one testicular seminoma and 70\% of clinical stage one NSGCT (8).

This article intends to provide an up-to-date summary of RO for the management of testicular cancer. We describe diagnostic and pre-operative considerations, gold standard surgical technique, new developments and variations, and post-operative considerations. 
Table 1 Differential diagnoses of testicular masses which can be generally determined based on history, examination and ultrasound findings (11,13-16)

\begin{tabular}{lll}
\hline Testicular masses & Benign & Malignant \\
\hline Intra-testicular & $\begin{array}{l}\text { Epidermoid cysts; tunica albuginea cysts; tubular ectasia of rete } \\
\text { testes; haematoma; infarction; orchitis }\end{array}$ & Seminoma; NSGCT; sex cord/stromal tumour; \\
& PTL & \\
Extra-testicular & Hydrocele; spermatocele; varicocele; inguinal hernia; spermatic & Spermatic cord sarcoma; epididymal sarcoma \\
& $\begin{array}{l}\text { cord lipoma; epididymitis; torsion; post-vasectomy sperm } \\
\text { granuloma; epididymal cystadenoma; adenomatoid scrotal }\end{array}$ & \\
& tumour & \\
\hline
\end{tabular}

NSGCT, non-seminomatous germ cell tumour; PTL, primary testicular lymphoma.

\section{Pre-operative considerations}

\section{Diagnosis}

\section{Clinical presentation}

Testicular cancer typically presents as a painless unilateral scrotal mass identified on self-examination, by a sexual partner, or as an incidental ultrasound finding. Acute scrotal pain is present in an estimated $20 \%$, is caused by tumour haemorrhage or infarction, and can result in misdiagnosis of epididymo-orchitis or torsion $(8,9)$. Uncommon presentations include gynaecomastia in $7 \%$, and manifestations of metastatic disease including weight loss, back/flank pain or palpable nodal disease (abdomen, neck) in $11 \%(10)$.

\section{Ultrasound imaging}

American Urological Association (AUA) and European Association of Urology (EAU) 2019 guidelines recommend prompt physical examination followed by imaging with testicular ultrasound (US) in any patient with suspicion of testicular cancer $(6,7)$. US has a sensitivity and specificity for malignancy of almost $100 \%$, distinguishes intraparenchymal from extra-testicular masses, differentiates malignant and inflammatory lesions, and can evaluate the contralateral testis $(11,12)$. US plays a pivotal role in assessing the many potential differential diagnoses when considering a testicular mass, as described below and summarised in Table 1 .

\section{Differential diagnoses}

Careful consideration should be given to the potential differential diagnoses of a testicular mass, as the underlying aetiology can impact the surgical approach taken. For example, a benign extra-testicular mass such as a hydrocele can be managed using a trans-scrotal technique, whilst any suspected malignant mass should be approached with an inguinal exploration.
Masses identified as extra-testicular or para-testicular on examination or US are generally benign in aetiology, with potential diagnoses including hydrocele, spermatocele, varicocele, and inguinal hernia with scrotal extension $(13,14)$. Painful testicular masses are also chiefly benign, and either inflammatory or traumatic in origin including epididymo-orchitis, torsion and haematoma (13). These generalisations should be approached with caution with careful consideration of history, examination and US findings together crucial-20\% of testicular malignancies present with pain secondary to tumour haemorrhage or infarction, and concurrent hydrocele can potentially mask an underlying malignant mass (9). Spermatic cord sarcoma whilst rare, accounts for the majority of malignant paratesticular tumours and are usually clinically distinct from the testis (15).

More than $95 \%$ of intra-testicular masses are malignant, and frequently demonstrate a solid hypoechoic appearance with internal vascularity on US $(11,13)$. Benign cystic intraparenchymal lesions are rare, but may include tubular ectasia of the rete testes, epidermoid cysts, and tunica albuginea cysts. Solid intraparenchymal malignant lesions are seminoma or non-seminomatous GCT in the majority, with the former generally homogenous and the latter more heterogenous on US (11).

Primary testicular lymphoma (PTL) is an important differential diagnosis to consider particularly in older men $>60$ years old and men with bilateral masses, as it is the commonest testicular malignancy in these groups (17). The majority of PTL is diffuse large B-cell on histology, but secondary involvement of the testis with systemic lymphoma does occasionally occur (11). Often PTL is diagnosed on histopathology from RO, but even when it is clinically suspected; inguinal RO remains the gold standard for initial treatment to achieve optimal disease control and provide an adequate specimen for immunohistochemistry evaluation (17). 
The blood-testis barrier reduces chemotherapy penetration, making local disease control crucial and contributing to the contralateral testis being a common site of relapse in PTL $(18,19)$. Management is then guided by extranodal lymphoma guidelines rather than testicular GCT, and includes chemoradiotherapy.

\section{Serum tumour markers}

Serum tumour marker testing of alpha-fetoprotein (AFP), human chorionic gonadotropin (hCG), and lactate dehydrogenase (LDH) is imperative prior to RO. This is to aid diagnosis of testicular masses, and facilitate accurate staging which helps predict prognosis of GCT following $\mathrm{RO}(6,7)$.

AFP is elevated in up to $40 \%$ of early stage NSGCT including yolk sac tumours and embryonal carcinoma, but is not excreted by choriocarcinoma or seminoma (20). Elevated hCG is seen in up to $30 \%$ of low stage NSGCT and $10-15 \%$ of seminoma, as it is secreted by choriocarcinoma, embryonal carcinoma, and syncytiotrophoblastic cells that are occasionally seen in seminoma (20). $\mathrm{LDH}$ is the least specific serum tumour marker for GCT, is elevated in $20 \%$ of all early stage GCT, and is more useful for prognosis than diagnosis (20).

\section{Radiological staging}

Radiological imaging is used to evaluate regional and distant lymph node and visceral metastasis. American and European guidelines recommend computed tomography (CT) of the chest, abdomen and pelvis in all newly diagnosed GCT given the earliest site of lymphatic dissemination is frequently the retroperitoneal lymph nodes (RLN), and the first site of visceral metastasis is often lung $(6,7)$. Staging CT scan await pathological confirmation of testicular malignancy or be performed pre-operatively if the diagnosis is clear.

\section{Fertility counselling}

Given testicular cancer is generally a disease of younger fertile men, it is crucial to consider fertility prior to performing RO. Guidelines now include strong recommendations to counsel patients about semen cryopreservation prior to surgery and perform preoperative fertility assessment, given the risk of subfertility or infertility that is associated with testicular GCT and its surgical and adjunct treatments $(6,7)$. Sperm banking prior to $\mathrm{RO}$ is even more crucial in men of reproductive age with an abnormal contralateral testis or known subfertility, given the risk of azoospermia following $\mathrm{RO}$ in this group (7).

\section{Testicular prosthesis}

All patients planned for inguinal exploration with potential RO, should be pre-operatively counselled regarding the possibility of elective testicular prosthesis at time of surgery $(6,7)$. Testicular prosthesis insertion at time of RO has been demonstrated to be safe (21), and is associated with high rates of satisfaction (22). Although risk is low, patients should be informed of potential complications of the prosthesis including infection, malposition and need for replacement or removal.

\section{Informed consent}

Following appropriate diagnostic work-up and preoperative counselling as described above, informed consent should be obtained for inguinal exploration with RO if testicular malignancy is suspected.

RO performed via an inguinal approach should be described as the gold standard for oncological control in the majority of patients $(6,7)$. It not only establishes diagnosis and facilitates clinical staging, but is also curative for $>80 \%$ of men with clinical stage one testicular seminoma and $70 \%$ of clinical stage one NSGCT (8). Testis sparing surgery (TSS) and surveillance should be described as alternative approaches only in specific scenarios and risk of disease progression or recurrence should be discussed $(6,7)$.

Risks of complications associated with RO should be discussed and are summarised in Table 2.

\section{Radical inguinal orchidectomy: surgical technique}

The surgical technique for radical inguinal orchidectomy (RO) is well described, any variances tend to relate to surgeon preference, and is underpinned by two principles relating to oncological control $(6,7,23,26-28)$ :

(I) Inguinal exploration is necessary with the testis exteriorised within its tunica vaginalis;

(II) The spermatic cord should be ligated at the level of the internal inguinal ring for adequate oncological control and to facilitate easy identification if further surgery with retroperitoneal lymph node dissection (RPLND) is required. 
Table 2 Risks and complications associated with RO that should be discussed when obtaining informed consent (23-25)

\begin{tabular}{lll}
\hline Risk/complication & Incidence & Subsequent issues \\
\hline Scrotal haematoma & $1-2 \%$ & Rarely requires surgical intervention but may take weeks to resolve \\
Infection & $1 \%$ & Rarely requires intervention \\
Postoperative pain & $60 \%$ initially; 1.8\% 1 year post-op & Higher rates of phantom testis syndrome \\
Phantom testis syndrome & $25 \%$ & $\begin{array}{l}\text { Usually begins }>2 \text { mth post-op; Triggered by urination, ejaculation or } \\
\text { exercise in } 40 \% \text {; Can be chronic in } 25 \%\end{array}$ \\
Ilioinguinal nerve injury & Rare & $\begin{array}{l}\text { Can cause chronic pain and paraesthesia of superior medial thigh and } \\
\text { anterior scrotum }\end{array}$ \\
Inguinal hernia & $<1 \%$ & $\begin{array}{l}\text { May require subsequent hernia repair } \\
\text { Tumour spillage }\end{array}$ \\
Reduced fertility & Rare & Requires post-operative chemotherapy \\
Hypogonadism & $20 \%$ oligospermic; $5 \%$ azoospermic & Rates of oligospermia and azoospermia higher if pre-existing subfertility \\
& $0.5 \% 1$ year post-op & $\begin{array}{l}\text { Subfertility and metabolic consequences such as weight gain, } \\
\text { hypercholesterolaemia, and hypertension; may require adjuvant }\end{array}$ \\
\hline
\end{tabular}

\section{Preparation and positioning}

The correct side should be confirmed by the patient and US images, and marked, surgical time-out performed, and a general anaesthetic administered. Regional anaesthetic is possible but rarely used due to short duration of the procedure, and potential reflex response when traction to the testicle and spermatic cord is applied (28). The patient is positioned supine, the ipsilateral inguinal and lower abdominal area shaved and cleaned with a surgical scrub, and a square sterile drape positioned to expose the ipsilateral inguinal region and hemiscrotum with the penis pulled away from the surgical field.

\section{Incision}

A traditional oblique incision should start $2 \mathrm{~cm}$ superior and lateral to the pubic tubercle, and then be extended toward the superior anterior iliac spine along a Langer line parallel to the inguinal ligament $(23,26-28)$. The recommended length of incision varies $(3-10 \mathrm{~cm})$ but should be guided by the size of the testicular mass, and ability to access the internal or deep ring for ligation (24,28-30). An alternative more horizontal incision that extends $5-8 \mathrm{~cm}$ from one finger breadth above the external inguinal ring, laterally toward the internal inguinal ring is also possible (28).

The inguinal incision can be extended toward the scrotum to facilitate delivery of a large testicular tumour and should be considered in this circumstance to avoid testis rupture and tumour spillage $(23,26)$.

\section{Exposure}

The incision is deepened using a scalpel or diathermy, to reach the external oblique (EO) aponeurosis, being mindful of iliolumbar or deep circumflex iliac veins that accompany the arteries supplying the EO (29), and require ligation. Once the EO aponeurosis is adequately exposed and cleaned of subcutaneous fat, it is sharply incised to open the roof of the inguinal canal. This incision should extend medially to the external inguinal ring and laterally to a point overlying the internal ring, which is approximately $4 \mathrm{~cm}$ in length (27).

It is at this point the ilioinguinal nerve is identified on the anterior surface of the spermatic cord and carefully dissected free from the external spermatic fascia and cremasteric muscle that invest it (26). It is recommended by all authors that the ilioinguinal nerve then be cautiously retracted out of the surgical field to avoid injury during dissection of the cord which can result in chronic postoperative pain and paraesthesia of the upper thigh and anterior scrotum $(24,26-28)$.

\section{Dissection of spermatic cord}

The spermatic cord is now gently mobilised at the level of the pubic tubercle using blunt dissection to develop the plane between the cord and the floor of the inguinal canal, 
until the cord can be encircled with thumb and forefinger $(26,28)$. Inadvertent dissection through the floor of the inguinal canal should be avoided to reduce the risk of postoperative direct inguinal hernia (26).

Once the spermatic cord is adequately mobilised, it is encircled twice with a small Penrose drain to create a tourniquet effect, and secured with a clamp just distal to the internal inguinal ring (26-28). This provides early vascular control and allows traction of the cord to facilitate delivery of the testis.

\section{Delivery of the testicle}

Whilst applying gentle traction to the spermatic cord in a cephalad direction and external pressure to the ipsilateral hemiscrotum, the testis within its tunica vaginalis is gently pushed into the surgical field (26-28). Further dissection is often required to mobilise the tunica vaginalis from its investing fascial layers (26).

The inguinal incision can be extended toward the scrotum to facilitate delivery of a large testicular tumour and should be considered in this circumstance to avoid testis rupture and tumour spillage $(23,26)$. Copious wash with distilled water and postoperative chemotherapy is required in the case of tumour spillage (23).

Once the testicle has been delivered into the surgical field, the gubernaculum if present, is divided at the most inferior aspect of the testis. Any vessels identified within the gubernaculum can be ligated with ties or diathermy. The testis within its tunica vaginalis is now free and attached only by the spermatic cord (26-28).

\section{Ligation of spermatic cord}

The spermatic cord is now mobilised to the level of the internal inguinal ring. Both Hinman and Campbell-Walsh favour further dissection of the cord proximally, to visualise the peritoneal reflection at the point where the vas deferens diverges from the spermatic vessels $(26,27)$. This proximal dissection for high cord ligation gives oncological control, and facilitates completion resection of the abdominal spermatic cord if subsequent RPLND is required, as the cord structures can retract inside the retroperitoneal space (23).

A triple-clamp technique is suggested to divide the spermatic cord, with two clamps placed at the level of the internal ring proximal to the Penrose tourniquet, and a third clamp distal to the tourniquet. The cord can then be transected, the testicle and attached spermatic cord removed, and non-absorbable sutures applied to the divided cord (28). Suture ligation is recommended to avoid ties slipping off the retracting proximal spermatic cord, as subsequent intra-abdominal bleeding may require a laparotomy to secure the bleeding vessels.

Although the spermatic cord can be ligated as one structure, for bulky cords it is possible to dissect the vas deferens from the gonadal vessels, and divide them separately $(26,27)$. Individually ligating the vas deferens from the remainder of the cord structures facilitates easy retrieval of the spermatic cord stump during RPLND where the vas deferens is not taken and helps to minimise risk of scrotal or retroperitoneal haematoma $(27,28)$.

\section{Haemostasis and closure}

Following meticulous haemostasis and copious irrigation, a testicular prosthesis can be placed in the empty testicular fossa if consented for pre-operatively (28). A common technique involves inserting a Foley catheter into the most dependant part of the scrotum, with the volume needed to inflate the balloon used to estimate the size of the prosthesis required; then the scrotal skin is inverted and the prosthesis is secured to the dartos with a single suture (30). This technique prevents a 'high-riding' prosthesis and facilitates correct sizing for good cosmesis.

The EO aponeurosis is then closed with a running absorbable suture, with caution taken to avoid the ilioinguinal nerve which should be carefully placed on the floor of the inguinal canal (27). Routine closure of the subcutaneous fascial layers and skin completes the procedure.

\section{Other considerations for orchidectomy}

\section{Scrotal orchidectomy}

International guidelines strongly advise against a transscrotal approach for any testicular mass suspicious for malignancy $(6,7)$. Scrotal violation is associated with a significantly higher risk of local recurrence when compared with RO, though surprisingly no difference in rates of metastatic disease is demonstrated (31).

Scrotal violation tends to occur with the unexpected intra-operative finding of a testis tumour during elective scrotal surgery. In this scenario where scrotal orchidectomy is performed, patients should be counselled that the risk of local recurrence is $2.5 \%$ which is higher than those 
having inguinal $\mathrm{RO}$, and that rarely they may be considered for adjunctive therapy with scrotal scar excision and/or radiotherapy but that this is not mandated (7).

A modified sub-inguinal orchidectomy to avoid the morbidity related to high ligation of the cord has been proposed, with overall survival and disease free outcomes not affected by spermatic cord invasion (32). This knowledge is certainly beneficial to those having an unplanned scrotal orchidectomy where proximal cord ligation cannot be performed.

A trans-scrotal approach for simple orchidectomy can be performed electively for hormonal ablation in advanced prostate cancer (27).

\section{RO for para-testicular sarcoma}

Para-testicular tumours are rare, and when malignant are almost always sarcoma (15). A high index of suspicion is required as in a recent analysis of 51 patients with paratesticular sarcoma, $>90 \%$ underwent initial surgery without a pre-operative diagnosis of sarcoma. There is a high risk of local recurrence if adequate margins are not taken (33).

In patients with a high index of suspicion, US and magnetic resonance imaging (MRI) followed by percutaneous biopsy will help diagnose para-testicular sarcoma, and multi-disciplinary discussion is mandatory given its rarity. Neoadjuvant radiotherapy is often used to improve margin status, but wide-margin hemiscrotectomy at initial surgery or as a completion or salvage procedure, is also reported to have favourable oncological outcomes (33) and avoid radiation injury to the contralateral testis.

\section{TSS}

\section{Rationale}

Partial orchidectomy (PO), or TSS, is being increasingly accepted as a more conservative alternative for smaller testicular masses. Some series suggest that $60-70 \%$ of nonpalpable masses $<20 \mathrm{~mm}$ are non-malignant lesions such as small benign Leydig or Sertoli cell tumours (34-37). Preservation of hormonal function and fertility is also a potential advantage of TSS, particularly in men with bilateral synchronous lesions or solitary testis.

International guidelines recommend TSS be considered as an alternative to $\mathrm{RO}$ in selected patients with high probability of a benign tumour $(<20 \mathrm{~mm}$, equivocal ultrasound, nonpalpable, negative serum tumour markers), or an anatomical or functional solitary testis where preserved gonadal function is desired, or bilateral synchronous tumours $(6,7)$.

\section{Surgical technique}

The approach to $\mathrm{PO}$, with delivery of the testicle into the surgical field, is similar to RO. There is debate as to the necessity of ischaemia and if hypothermia is required. Traditionally, manipulation is performed under cold ischaemia with the spermatic cord clamped or occluded with the Penrose tourniquet $(38,39)$. Cold ischaemia is achieved by immersion of the testis in an ice slush solution for 5-10 minutes after spermatic cord clamping (36). Giannarini et al., reports many urologists have abandoned ischaemia to preserve vascularisation and optimal gonadal function given the indications for the testis sparing approach (36). Whilst most authors still recommend division of the gubernaculum $(36,38,39)$, a retrospective series of 65 patients described a modern technique without both ischaemia and spermatic cord clamping, and leaving the gubernaculum intact to avoid postoperative malpositioning of the testis (40).

Once exteriorised, the testis should be placed on separate sterile drapes to avoid potential tumour spillage and wound contamination $(27,36)$. The tunica vaginalis is opened anteriorly, and intraoperative ultrasound is recommended to reliably localise the tumour. A scalpel is used to incise the tunica albuginea overlying the mass $(27,36,38)$. Ultrasound guided stereotactic enucleation has been described (41), though should be considered experimental $(36,37)$.

Once the testicular mass is exposed, it should be enucleated with a rim of adjacent parenchyma gently scraped away, as small testicular tumours frequently exhibit a pseudocapsule $(27,38)$. Following enucleation, it is recommended multiple (generally four) biopsies be taken of the surrounding normal testicular parenchyma $(6,7)$, as presence of in situ germ cell neoplasia is associated with increased recurrence and adjuvant radiation therapy may need to be considered (37).

Frozen section examination (FSE) is recommended in the AUA guidelines as part of TSS. The approach to managing the remnant testicle if FSE is positive for testicular GCT should be discussed pre-operatively, with patients counselled about the higher risk of local recurrence and need for adjuvant radiation therapy if $\mathrm{RO}$ is not performed (7).

Following meticulous haemostasis, each layer of the tunica is closed with an absorbable suture, the testicle is placed back into the dependent part of the scrotum ensuring 
no twisting of the spermatic cord, and the subcutaneous layers and skin are closed $(27,38)$.

\section{Utilisation}

Alanee et al., performed a large scale review of primary testicular cancer treatment between 1995-2011; and found that of 5,365 patients identified as having small testicular tumours that would be amenable to PO, only $2 \%$ had TSS performed (42). No difference was demonstrated in cancer specific survival between the RO and PO groups in the 5,365 patients with tumours $\leq 2 \mathrm{~cm}$ (42). Low utilisation of PO was also demonstrated in 81 patients with tumours $<10$ $\mathrm{mm}$ in size by Scandura et al., with only $5 \%$ undergoing PO rather than RO despite $69 \%$ of these cases being identified as benign at histopathology (35).

Despite well described indications and technique of $\mathrm{PO}$, and its inclusion for consideration in international guidelines, there remains minimal uptake of this approach. Limiting factors likely include lack of urological experience with the procedure, need for an experienced pathologist to interpret the FSE, and presence of a normal contralateral testis.

\section{Delayed orchidectomy}

Orchidectomy whether radical or partial, plays a pivotal role in the diagnostic algorithm for testicular cancer in the majority of patients, and is therapeutic in those with clinical stage one disease. In the small group of patients who present with symptomatic disseminated disease without an obvious testicular mass, diagnostic serum tumour markers and biopsy of distant disease are often enough to guide upfront chemotherapy which can be life-saving (6).

Subsequent testicular US will often demonstrate a small mass or focal calcification consistent with a 'burned out' GCT where the primary malignancy is thought to outgrow its blood supply, resulting in regression of the tumour (11). In these patients, delayed RO is preferred $(6,27)$.

\section{New developments \& future directions}

\section{Spermatic cord sparing orchidectomy}

Haroon et al., recently published an eight-year retrospective review of RO analysing spermatic cord invasion which was present in 3/121 (4\%) of cases (32). After reviewing the potential complications of $\mathrm{RO}$, the authors raised the question of whether avoiding opening the $\mathrm{EO}$ and clamping the spermatic cord with a sub-inguinal approach could be feasible and safe. The overall and disease free 5 -year survival of these 3 patients was not affected by spermatic cord invasion, leading to Haroon et al., nominating a modified sub-inguinal orchidectomy as a potentially viable and less morbid procedure in clinical stage 1 testicular GCT (32). This requires prospective long-term studies to assess oncological outcomes and post-operative complications, but presents a potential shift in a technique that has been previously described as 'timeless' (26).

\section{Surveillance for small testicular tumours}

Another area requiring further exploration is whether surgery can be avoided altogether in small tumours $<10 \mathrm{~mm}$ in size. Scandura et al., recently identified 81 cases in a cohort of $>2,600$ patients where tumour size was $<10 \mathrm{~mm}$, where $69 \%$ were benign and yet $95 \%$ underwent RO (35). The authors suggest that patients with lesions $5-10 \mathrm{~mm}$ who undergo RO should be counselled that two thirds are benign, and patients with lesions $<5 \mathrm{~mm}$ should avoid surgery altogether and instead have close surveillance with ultrasound (35). Larger scale prospective studies evaluating the use of testicular biopsy and/or PO versus close surveillance in the cohort of tumours $<10 \mathrm{~mm}$ should be considered to confirm whether this represents a viable and safe future alternative.

\section{Conclusions}

Radical inguinal orchidectomy with division of the spermatic cord at the internal inguinal ring is the gold standard for diagnosis and local treatment of testicular malignancies. As alluded to, technique has not dramatically changed and there is a wide-ranging consensus between textbooks, articles and guidelines as to the recommended approach.

Careful pre-operative work up with consideration of benign lesions, indications for potential TSS, and percutaneous biopsy for suspected para-testicular sarcoma can avoid potential intra-operative uncertainty at radical inguinal orchidectomy.

\section{Acknowledgments}

Funding: None. 


\section{Footnote}

Provenance and Peer Review: This article was commissioned by the Guest Editor (Shomik Sengupta) for the series "Surgery for Urologic Cancers" published in Translational Andrology and Urology. The article has undergone external peer review.

Conflicts of Interest: The series "Surgery for Urologic Cancers" was commissioned by the editorial office without any funding or sponsorship. The authors have no conflicts of interest to declare.

Ethical Statement: The authors are accountable for all aspects of the work in ensuring that questions related to the accuracy or integrity of any part of the work are appropriately investigated and resolved.

Open Access Statement: This is an Open Access article distributed in accordance with the Creative Commons Attribution-NonCommercial-NoDerivs 4.0 International License (CC BY-NC-ND 4.0), which permits the noncommercial replication and distribution of the article with the strict proviso that no changes or edits are made and the original work is properly cited (including links to both the formal publication through the relevant DOI and the license). See: https://creativecommons.org/licenses/by-nc-nd/4.0/.

\section{References}

1. La Vecchia C, Bosetti C, Lucchini F, et al. Cancer mortality in Europe, 2000-2004, and an overview of trends since 1975. Ann Oncol 2010;21:1323-60.

2. Siegel RL, Miller KD, Jemal A. Cancer statistics, 2019. CA Cancer J Clin 2019;69:7-34.

3. Nigam M, Aschebrook-Kilfoy B, Shikanov S, et al. Increasing incidence of testicular cancer in the United States and Europe between 1992 and 2009. World J Urol 2015;33:623-31.

4. Powles TB, Bhardwa J, Shamash J, et al. The changing presentation of germ cell tumours of the testis between 1983 and 2002. BJU Int 2005;95:1197-200.

5. Algaba F, Sesterhenn IA. Chapter 1: Histological Classification and Pathology of Testicular Tumors. In: Laguna MP, Albers P, Bokemeyer C, et al. editors. Cancer of the Testis. 1st edition. Berlin: Springer-Verlag, 2010:3-26.

6. Laguna MP, Albers P, Albrecht W, et al. EAU Guidelines on Testicular Cancer 2019. European Association of
Urology Guidelines 2019 Edition. Presented at the EAU Annual Congress Barcelona 2019. ed. Arnhem, The Netherlands: European Association of Urology Guidelines Office, 2019.

7. Stephenson A, Eggener SE, Bass EB, et al. Diagnosis and Treatment of Early Stage Testicular Cancer: AUA Guideline. Practice Guideline J Urol 2019;202:272-81.

8. Goldberg H, Klaassen Z, Chandrasekar T, et al. Germ Cell Testicular Tumors-Contemporary Diagnosis, Staging and Management of Localized and Advanced disease. Urology 2019;125:8-19.

9. Stevenson SM, Lowrance WT. Epidemiology and Diagnosis of Testis Cancer. Urol Clin North Am 2015;42:269-75.

10. Moul JW. Timely diagnosis of testicular cancer. Urol Clin North Am 2007;34:109-17; abstract vii.

11. Coursey Moreno C, Small WC, Camacho JC, et al. Testicular tumors: what radiologists need to know-differential diagnosis, staging, and management. Radiographics 2015;35:400-15.

12. Barrisford GW, Kreydin EI, Preston MA, et al. Role of imaging in testicular cancer: current and future practice. Future Oncol 2015;11:2575-86.

13. Smith ZL, Werntz RP, Eggener SE. Testicular Cancer: Epidemiology, Diagnosis, and Management. Med Clin North Am 2018;102:251-64.

14. Bernard B, Sweeney CJ. Diagnosis and Treatment of Testicular Cancer: A Clinician's Perspective. Surg Pathol Clin 2015;8:717-23.

15. Moschini M, Mattei A. Diagnosis and management of spermatic cord tumors. Curr Opin Urol 2017;27:76-9.

16. Khoubehi B, Mishra V, Ali M, et al. Adult paratesticular tumours. BJU Int 2002;90:707-15.

17. Cheah CY, Wirth A, Seymour JF. Primary testicular lymphoma. Blood 2014;123:486-93.

18. Kemal Y, Teker F, Demirag G, et al. Primary testicular lymphoma: a single centre experience. Exp Oncol 2015;37:223-6.

19. Bart J, Groen HJ, van der Graaf WT, et al. An oncological view on the blood-testis barrier. Lancet Oncol 2002;3:357-63.

20. Gilligan TD, Seidenfeld J, Basch EM, et al. American Society of Clinical Oncology Clinical Practice Guideline on uses of serum tumor markers in adult males with germ cell tumors. J Clin Oncol 2010;28:3388-404.

21. Robinson R, Tait CD, Clarke NW, et al. Is it safe to insert a testicular prosthesis at the time of radical orchidectomy for testis cancer: an audit of 904 men undergoing radical 
orchidectomy. BJU Int 2016;117:249-52.

22. Clifford TG, Burg ML, Hu B, et al. Satisfaction With Testicular Prosthesis After Radical Orchiectomy. Urology 2018;114:128-32.

23. Pizzocaro G, Guarneri A. Inguinal orchidectomy for testicular cancer. BJU Int 2009;103:704-16.

24. Wren J, Brannigan R. Chapter 53: Complications of Surgery of the Testicle, Vas Deferens, Epididymis and Scrotum. In: Taneja SS, Shah O, editors. Taneja's Complications of Urologic Surgery: Diagnosis, Prevention and Management. 5th edition. Amsterdam: Elsevier, 2018:567-79.

25. Wiechno PJ, Kowalska M, Kucharz J, et al. Dynamics of hormonal disorders following unilateral orchiectomy for a testicular tumor. Med Oncol 2017;34:84.

26. Elmajian DA. Chapter 117: Radical Orchiectomy. In: Smith J, Howards S, Preminger G, et al. editors. Hinman's Atlas of Urologic Surgery. 4th edition. Amsterdam: Elsevier, 2016:840-2.

27. Rice KR, Cary CK, Masterson TA, et al. Chapter 35: Surgery of Testicular Tumors. In: Wein AJ, Kavoussi LR, Partin AW, et al. editors. Campbell-Walsh Urology. 11th edition. Amsterdam: Elsevier, 2015:815-37.

28. Lee JD, Scherr DS. Chapter 59: Radical Inguinal Orchiectomy. In: Keane TE, Graham SD, editors. Glenn's Urologic Surgery. 8th edition. Philadelphia: Lippincott Williams \& Wilkins, 2015:528-32.

29. Schlenz I, Burggasser G, Kuzbari R, et al. External oblique abdominal muscle: a new look on its blood supply and innervation. Anat Rec 1999;255:388-95.

30. Simms MS, Huq S, Mellon JK. Testicular prostheses: a new technique for insertion. BJU Int 2004;93:179.

31. Capelouto CC, Clark PE, Ransil BJ, et al. A review of scrotal violation in testicular cancer: is adjuvant local therapy necessary? J Urol 1995;153:981-5.

32. Haroon UM, Bhatt NR, Akram CM, et al. Significance of

Cite this article as: Koschel SG, Wong LM. Radical inguinal orchidectomy: the gold standard for initial management of testicular cancer. Transl Androl Urol 2020;9(6):3094-3102. doi: 10.21037/tau.2019.12.20 spermatic cord invasion in radical inguinal orchidectomy: Will a modified subinguinal orchidectomy suffice? Urologia 2020;87:70-4.

33. Goldberg H, Wong LM, Dickson B, et al. Long-term oncological outcomes of patients with paratesticular sarcoma. BJU Int 2019;124:801.

34. Bojanic N, Bumbasirevic U, Bojanic G, et al. Testis sparing surgery for treatment of small testicular lesions: Is it feasible even in germ cell tumors? J Surg Oncol 2017;115:287-90.

35. Scandura G, Verrill C, Protheroe A, et al. Incidentally detected testicular lesions $<10 \mathrm{~mm}$ in diameter: can orchidectomy be avoided? BJU Int 2018;121:575-82.

36. Giannarini G, Dieckmann KP, Albers P, et al. Organsparing surgery for adult testicular tumours: a systematic review of the literature. Eur Urol 2010;57:780-90.

37. Djaladat H. Organ-sparing surgery for testicular tumours. Curr Opin Urol 2015;25:116-20.

38. Heidenreich A. Chapter 60: Organ-Preserving Surgery in Testicular Tumors. In: Keane TE, Graham SD, editors. Glenn's Urologic Surgery. 8th edition. Philadelphia: Lippincott Williams \& Wilkins, 2015:533-7.

39. Tanrikut C, Goldstein M. Chapter 116: Testis-Sparing Surgery for Benign and Malignant Tumors. In: Smith J, Howards S, Preminger G, et al. editors. Hinman's Atlas of Urologic Surgery. 4th edition. Berlin: Elsevier, 2016:836-9.

40. Leonhartsberger N, Pichler R, Stoehr B, et al. Organ preservation technique without ischemia in patients with testicular tumor. Urology 2014;83:1107-11.

41. Hopps CV, Goldstein M. Ultrasound guided needle localization and microsurgical exploration for incidental nonpalpable testicular tumors. J Urol 2002;168:1084-7.

42. Alanee S, Clemons J, Holland B, et al. The Utilization of Partial Orchiectomy in Treating Small Testicular Tumors in the United States. Int J Cancer Clin Res 2017;4:84. 